\title{
Quelques aspects de la détection des graisses étrangères dans la matière grasse butyrique
}

\author{
par \\ H. HENDRICKX et A. HUYGHEBAERT \\ Université de Gand, Faculté des Sciences Agronomiques, \\ Laboratoire de Science laitière, de Technologie des Produits laitiers \\ et des Graisses alimentaires \\ Coupure, 533 Gand (Belgique)
}

\section{Introduction}

La détection des graisses étrangères dans la matière grasse butyrique est un problème très complexe. Cette graisse contient en effet une série très étendue de composants majeurs et un nombre très élevé de composants mineurs ; par conséquent il est très difficile de trouver une propriété qu'on ne rencontre pas dans la graisse butyrique. D'autre part en dehors des situations économiques anormales le prix de cette graisse est très élevé ; on doit donc en principe considérer toutes les autres graisses alimentaires comme agent de falsification possible.

Il existe des publications très intéressantes sur ce problème, de Roos [1] et de Guillaumin [2].

Nous avons abordé l'étude de la détection des graisses étrangères quand se présentaient, sur le marché, des graisses indiquées par la suite comme des graisses de substitution, dont les caractéristiques chimiques se rapprochaient de plus en plus de la matière grasse butyrique. La présence de ces graisses, préparées par interestérification de suif de bœuf, d'huile de coco ou de palmiste, de tributyrine et d'autres triglycérides synthétiques, a causé des problèmes très graves tant pour l'industrie laitière que pour les organisations de recherche et de contrôle. L'étude des possibilités de détection des graisses de substitution nous a amené à l'étude d'autres graisses de falsification.

Nous discuterons dans ce texte :

- les graisses de substitution,

- les graisses végétales,

- les graisses butyriques dénaturées,

- les graisses animales. 


\section{I. - Graisses de substitution}

Il s'agit des graisses préparées dans le but de remplacer la matière grasse butyrique ; ces graisses ont été modifiées en vue d'imiter le mieux que possible la matière grasse butyrique. Nous ne considérons donc pas comme graisse de substitution les graisses naturelles ou les mélanges de graisses naturelles, mais bien les graisses interestérifiées à base de suif de bœuf, huile de coco ou de palmiste (en vue d'obtenir une quantité suffisante d'acides gras à chaîne moyenne) et de triglycérides synthétiques comme la tributyrine (en vue d'augmenter la quantité d'acides gras à chaîne courte) ; dans certains cas on a utilisé aussi de la tricaprine dans le but de rendre normal le rapport $\mathrm{C} 12: 0 / \mathrm{C} 10: 0$. Pendant les années de recherche il $\mathrm{y}$ a eu une évolution remarquable dans la composition de ces graisses, qui, au point de vue ressemblance à la matière grasse butyrique, se sont rapprochées de plus en plus.

L'existence de ces graisses a été signalée dans plusieurs pays et par plusieurs auteurs, Hopper et al. [3], Guex [4], Bhalerao et Kummerow [5], Hansen et al. [6], Lück et Kon [7], Boniforti [8], De Francesco et Avancini [9], Guyot et Piraux [10], Roos et al. [11] et Parodi [12, 13].

Comme démontré dans le tableau 1, les méthodes classiques ne sont pas suffisantes pour déceler les graisses de substitution. Quand il existe des différences avec les valeurs normales pour le beurre, l'écart n'est pas assez grand pour permettre une base de détection.

L'examen par chromatographie en phase gazeuse permet de déterminer les acides gras individuels. A base de cette composition nous avons subdivisé les graisses de substitution en 4 types. Dans le tableau 2 ces valeurs sont comparées aux valeurs obtenues pour 235 échantillons de beurre belge. Les figures 1 et 2 montrent des chromatogrammes de la matière grasse butyrique et des graisses de substitution.

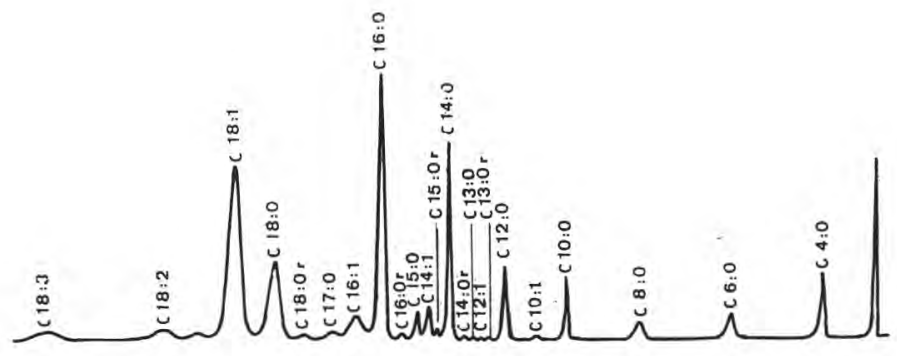

fig. 1

Chromatogramme des acides gras d'une matière grasse butyrique 
TABLEAU 1. - Les indices classiques des graisses de substitution et de la matière grasse butyrique

\begin{tabular}{l|r|r|r|r|r}
\hline \multirow{2}{*}{ Indice } & \multicolumn{2}{|c|}{ Graisse de substitution } & \multicolumn{1}{c}{$\begin{array}{c}\text { Matière grasse } \\
\text { butyrique }\end{array}$} \\
\cline { 2 - 5 } Reichert-Meissl & type 1 & type 2 & type 3 & type 4 & \\
\hline Polenske & $27,0-29,8$ & $23,7-27,8$ & $25,1-27,1$ & $32,7-34,3$ & $27,1-31,0$ \\
Xylol & $2,6-4,1$ & $1,8-4,5$ & $1,9-3,4$ & $0,9-1,6$ & $1,0-3,4$ \\
A & $21,3-23,9$ & $17,5-23,5$ & $21,9-24,7$ & $29,7-32,2$ & $17,3-21,1$ \\
B & $3,4-3,8$ & $1,8-4,7$ & $2,1-2,3$ & 1,6 & $4,5-9,6$ \\
Saponification & $35,3-36,9$ & $34,7-39,0$ & $37,1-38,1$ & 49,7 & $29,0-37,9$ \\
Réfraction & $224-230$ & $224-226$ & $218-227$ & $231-232$ & $222-236$ \\
Jode & $42,8-43,2$ & $43,1-43,8$ & $43,3-44,0$ & $42,6-42,7$ & $41,3-45,2$ \\
& $36,4-38,9$ & $35,8-39,6$ & $36,6-37,3$ & $38,5-40,1$ & $30,1-45,5$ \\
\hline
\end{tabular}




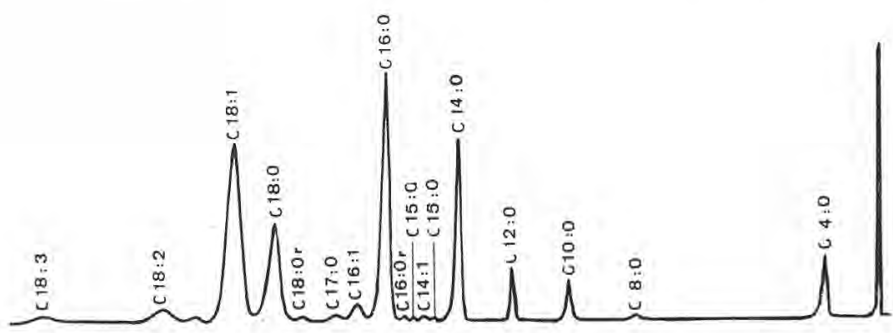

fig. 2

Chromatogramme des acides gras d'une graisse de substitution

Il est évident qu'il existe une conformité remarquable entre ces deux types de graisse ; néanmoins une différenciation est possible. L'absence des acides caproïque et caprylique dans les graisses de substitution ainsi que la disproportion dans les acides caprique et laurique dans certains types de ces graisses permettent de tirer des conclusions.

Des rapports entre les acides gras ont été proposés, pour la détection des graisses étrangères. De cette façon certains rapports e.a., les rapports $\mathrm{C} 4: 0 / \mathrm{C} 6: 0$ et $\mathrm{C} 4: 0 / \mathrm{C} 6: 0+\mathrm{C} 8: 0$ peuvent être très utiles. Le rapport $C 12: 0 / C 10: 0$ peut être appliqué pour le type 1 , mais est insuffisant pour les types 2 et 3 ; ce qui démontre l'évolution qui a eu lieu dans la composition de ces graisses. On peut conclure que les fabricants de ces graisses ont suivi les méthodes de détection et en même temps ont amélioré leurs produits.

Concernant l'emploi de ces rapports il faut néanmoins tenir compte des restrictions faites par Roos [14]. Il a démontré que l'utilisation d'un ou plusieurs rapports ne suffit pas pour démontrer certaines falsifications. Il faut aussi considérer les valeurs absolues ou - comme il a proposé - utiliser des ellipses de probabilité.

Comme il se produit toujours des fluctuations assez importantes dans la composition de la matière grasse butyrique (variations saisonnières...) nous avons recherché d'autres possibilités de détection des graisses de substitution.

En dehors de la composition en acides gras, il existe des possibilités dans la composition et la structure glycéridique. Nous avons mis en évidence (Hendrickx et Huyghebaert [15]), par chromatographie sur couche mince, la présence de monoglycérides dans les graisses de substitution. La différence nette entre ces graisses et la matière grasse butyrique est illustrée dans la figure 3. Ces monoglycérides se sont formés lors de la préparation de ces graisses, c'est-àdire l'interestérification. La teneur en monoglycérides, déterminée par la méthode de Jensen et Morgan [16] varie entre 1,52 et 2,95 $\mathrm{mmol} / 100 \mathrm{~g}$ de graisse. Il nous était possible par chromatographie 
TABLEAU 2. - La composition en acides gras des 4 types de graisse de substitution et de la matière grasse butyrique

\begin{tabular}{|c|c|c|c|c|c|}
\hline \multirow{2}{*}{ Acide gras } & \multirow{2}{*}{$\begin{array}{c}\text { Matière grasse } \\
\text { butyrique }\end{array}$} & \multicolumn{4}{|c|}{ Graisse de substitution } \\
\hline & & type 1 & type 2 & type 3 & type 4 \\
\hline $\begin{array}{ll}\text { C } & 4: 0 \\
\text { C } & 6: 0 \\
\text { C } & 8: 0 \\
\text { C } & 10: 0 \\
\text { C } & 10: 1 \\
\text { C } & 12: 0 \\
\text { C } & 13: 0 \mathrm{r} \\
\text { C } & 12: 1 \\
\text { C } & 13: 0 \\
\text { C } & 14: 0 \mathrm{r} \\
\text { C } & 14: 0 \\
\text { C } & 15: 0 \mathrm{r} \\
\text { C } & 14: 1 \\
\text { C } & 15: 0 \\
\text { C } & 16: 0 \mathrm{r} \\
\text { C } & 16: 0 \\
\text { C } & 16: 1 \\
\text { C } & 17: 0 \\
\text { C } & 18: 0 \mathrm{r} \\
\text { C } & 18: 0 \\
\text { C } & 18: 1 \\
\text { C } & 18: 2 \\
\text { C } & 18: 3\end{array}$ & $\begin{array}{c}3,4-4,1 \\
1,8-2,4 \\
0,9-1,5 \\
1,9-3,8 \\
0,2 \\
2,3-4,7 \\
\text { tr } \\
\text { tr } \\
0,1 \\
0,1 \\
8,5-13,4 \\
0,2 \\
1,1-1,9 \\
0,9-1,8 \\
0,2 \\
18,6-31,2 \\
1,9-3,6 \\
0,5 \\
0,2 \\
7,8-14,3 \\
23,0-34,5 \\
1,8-3,2 \\
0,9-4,1\end{array}$ & $\begin{array}{c}5,3-5,7 \\
\operatorname{tr}-0,1 \\
1,1-1,8 \\
0,9-1,1 \\
- \\
7,6-9,6 \\
- \\
- \\
\operatorname{tr} \\
\operatorname{tr} \\
4,7-5,7 \\
0,1 \\
0,1-0,2 \\
0,1-0,2 \\
0,1 \\
22,0-24,9 \\
2,5-3,1 \\
0,5 \\
0,2 \\
13,6-14,4 \\
32,1-32,5 \\
3,2-3,9 \\
0,7-1,1\end{array}$ & $\begin{array}{c}4,5-5,5 \\
\text { tr } \\
0,2-0,7 \\
2,2-3,0 \\
- \\
3,3-5,3 \\
- \\
- \\
\operatorname{tr} \\
\operatorname{tr} \\
9,3-12,6 \\
\operatorname{tr} \\
0,1-0,2 \\
0,1-0,2 \\
\operatorname{tr} \\
22,4-26,1 \\
2,8-3,2 \\
0,4 \\
0,2 \\
13,0-15,5 \\
25,5-32,6 \\
2,3-3,7 \\
0,8-1,2\end{array}$ & $\begin{array}{c}5,3-5,7 \\
\operatorname{tr} \\
0,2-0,3 \\
2,5-2,9 \\
- \\
2,7-2,9 \\
- \\
- \\
\operatorname{tr} \\
\operatorname{tr} \\
9,9-10,8 \\
\operatorname{tr} \\
0,1-0,2 \\
0,1-0,2 \\
\operatorname{tr} \\
23,0-23,6 \\
2,5-3,0 \\
0,5 \\
0,2 \\
13,2-15,5 \\
31,6-33,2 \\
3,0-3,6 \\
1,0-1,4\end{array}$ & $\begin{array}{c}5,9-6,5 \\
\operatorname{tr} \\
\operatorname{tr}-0,1 \\
0,2-3,2 \\
- \\
0,2-0,7 \\
- \\
- \\
\operatorname{tr} \\
\operatorname{tr} \\
3,9-10,9 \\
0,1-0,2 \\
0,6 \\
0,3-0,4 \\
0,1-0,3 \\
20,0-23,3 \\
3,3-4,6 \\
0,8 \\
0,3 \\
16,6-21,6 \\
33,6-34,1 \\
2,3-3,2 \\
0,5-0,9\end{array}$ \\
\hline
\end{tabular}


en couche mince de démontrer dans la graisse butyrique des quantités de l'ordre de 2,5 à 5 p. 100 des graisses de substitution. Les monoglycérides sont présents dans des quantités qu'on ne retrouve pas dans la graisse butyrique normale. Néanmoins, l'inconvénient de cette méthode est la formation de monoglycérides lors de la lipolyse. Une lipolyse peut se produire par exemple lors de la conservation du lait à la ferme à basse température par des lipases naturelles ou d'origine bactérienne ou même lors de la maturation des fromages. Cette action résulte dans une augmentation de l'acidité (teneur en acides gras libres) de la graisse butyrique.

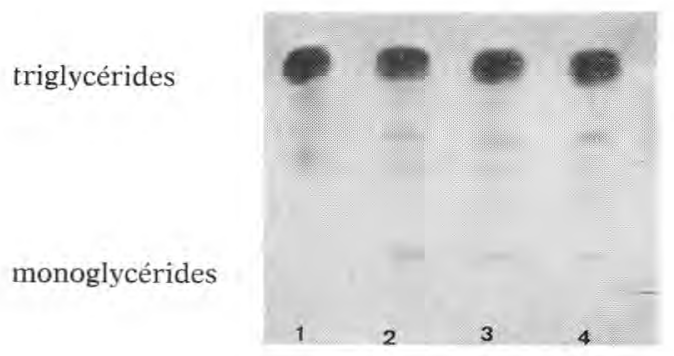

fig. 3

Chromatogramme sur couche mince des graisses de substitution et de la matière grasse butyrique :

1. matière grasse butyrique,

2. graisse de substitution (type 1),

3. graisse de substitution (type 2),

4. graisse de substitution (type 3).

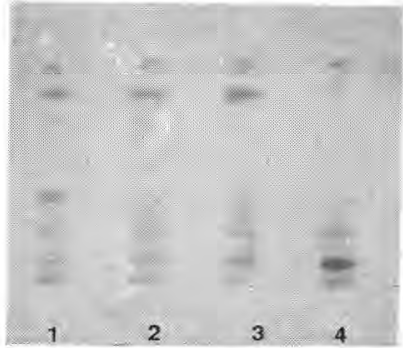

fig. 4

Chromatogramme en couche mince de l'insaponifiable des graisses de substitution et de la matière grasse butyrique :

1. graisse de substitution (type 1),

2. graisse de substitution (type 2),

3. graisse de substitution (type 3),

4. matière grasse butyrique,

Nous avons démontré qu'on peut appliquer la méthode de chromatographie en couche mince des monoglycérides sous réserve que l'acidité de la graisse butyrique n'est pas trop élevée, c'est-à-dire que nous n'avons pas pu démontrer des monoglycérides par la méthode élaborée dans \pm 300 échantillons de beurre de laiterie dont l'acidité variait entre 0,53 et 1,89.

Un troisième groupe de constituants qui s'est révélé important pour la détection des falsifications est l'insaponifiable. Il s'agit d'une série de substances qui sont présentes dans des quantités minimes. Parmi ces composants de l'insaponifiable il y a un groupe très connu : les stérols. Il existe une différence fondamentale entre les graisses d'origine animale qui contiennent du cholestérol et les graisses d'origine végétale qui contiennent des phytostérols.

L'examen de la composition des stérols des graisses de substitution nous a permis de formuler les conclusions suivantes : 
TABLEAU 3. - Composition des stérols majeurs des graisses de substitution et de la matière grasse butyrique

\begin{tabular}{|c|c|c|c|c|c|c|}
\hline \multirow{2}{*}{ Stérol } & \multirow{2}{*}{$\begin{array}{c}\text { Rétention } \\
\text { relative }\end{array}$} & \multicolumn{4}{|c|}{ Graisse de substitution } & \multirow{2}{*}{$\begin{array}{c}\text { Graisse } \\
\text { butyrique }\end{array}$} \\
\hline & & type 1 & type 2 & type 3 & type 4 & \\
\hline Cholestérol & 1,00 & 86,7 & 89,5 & 87,5 & 100,0 & 100,0 \\
\hline Brassicastérol & 1,12 & 1,8 & $\operatorname{tr}$ & 1,6 & - & - \\
\hline Campestérol & 1,32 & 1,2 & 2,0 & 2,5 & - & - \\
\hline Stigmastérol & 1,44 & 5,3 & 2,9 & 6,3 & - & - \\
\hline$\beta$-sitostérol & 1,68 & 4,7 & 5,6 & 2,1 & - & - \\
\hline
\end{tabular}


- la teneur en stérols est très basse : 0,04 à $0,08 \mathrm{p} .100$ par rapport à la matière grasse butyrique qui contient $\pm 0,30$ p. 100 de stérols,

- les stérols sont présents sous forme d'esters tandis que les stérols du beurre sont en majorité des stérols libres,

- la fraction stérolique, isolée par précipitation par la digitonine selon la norme FIL $n^{\circ} 32$ [17], est contaminée par des substances non stéroliques ; l'identification de ces composants sera discutée plus loin,

- la composition des stérols, déterminée par chromatographie en phase gazeuse (tab. 3) démontre que les types 1,2 et 3 contiennent des graisses d'origine végétale, qu'on ne retrouve plus dans le type 4 , Cette conclusion prouve de nouveau que la composition de ces graisses a été améliorée suivant les méthodes de détection appliquées.

Lors de l'examen par chromatographie sur couche mince de l'insaponifiable nous avons observé un groupe de substances que nous ne retrouvons pas dans la matière grasse butyrique (fig. 4). Par cette méthode il est également possible de démontrer des quantités minimales des graisses de substitution.

Plusieurs chercheurs se sont intéressés à ces composants inconnus dans l'insaponifiable des graisses de substitution. Guyot [18] les retrouvait dans la fraction stérolique lors de l'analyse par chromatographie gazeuse. Roos et al. [19] observaient que ces substances sont précipitées lors de l'isolation des stérols; ils désignaient ces composants comme insaponifiable insoluble. Nos travaux personnels ont été dirigés vers l'identification de ces composants. Nous avons démontré par chromatographie gazeuse, en couche mince et par spectroscopie infra-rouge que cette fraction est constituée de cétones et notamment de deux séries homologues du type $\mathrm{C}_{n} \mathrm{H}_{2 n+1} \mathrm{COC}_{n} \mathrm{H}_{2 n+1}$ et $\mathrm{C}_{2} \mathrm{H}_{2 n} \mathrm{COC}_{n} \mathrm{H}_{2 n-1}$ (Huyghebaert et al. [20].

Ces cétones, formées lors de la préparation des graisses de substitution, ne sont pas présentes dans la matière grasse butyrique.

En ce qui concerne les graisses de substitution, on peut conclure qu'il existe plusieurs possibilités de détection.

\section{II. - Les graisses d'origine végétale}

Quoique l'examen des acides gras permet de dépister des fraudes par des graisses végétales, la chromatographie en phase gazeuse des stérols est plus efficace et plus sensible. Plusieurs auteurs ont étudié ce problème et ont fait des propositions soit concernant l'isolation des stérols ou la préparation des stérols, soit concernant l'analyse elle-même ou l'interprétation des résultats, Roos et al. [19], La Croix [21], Guyot [18], Parodi [22], Kuzdzal-Savoie et Kuzdzal [23]. $\mathrm{La}$ teneur en stérols de la graisse butyrique varie entre 0,26 et 0,34 p. 100 , déterminée selon la norme FIL $n^{\circ} 32$ [17]. La teneur en 
stérols des graisses végétales est très variable. Ainsi la teneur en stérols de l'échantillon en question peut fournir des renseignements intéressants.

Lors de l'analyse par chromatographie gazeuse des stérols sous forme d'acétates nous retrouvons pour la graisse butyrique toujours 3 pics élués après le cholestérol (fig. 5). La présence d'une graisse végétale se traduit immédiatement par une augmentation du pic 3 ( $\beta$-sitostérol).

Comme dans le beurre non falsifié on détermine des petits pics qui ont le même temps de rétention que les phytostérols - pour lesquels on ne peut d'ailleurs pas conclure qu'il s'agit de phytostérols - on doit être prudent dans l'interprétation des résultats. Nous avons proposé (Hendrickx et Huyghebaert [24]) de calculer le rapport pic 3 / pic 2 et de conclure à une falsification quand ce rapport est $\geqslant 0,8$. La présence d'une graisse végétale peut être confirmée par le stigmastérol qu'on ne retrouve pas dans la graisse butyrique et qui se situe entre le pic 2 et le pic 3. Une autre possibilité est de déterminer le pourcentage du pic 3 ou du $\beta$-sitostérol comme proposé par Guyot [18].

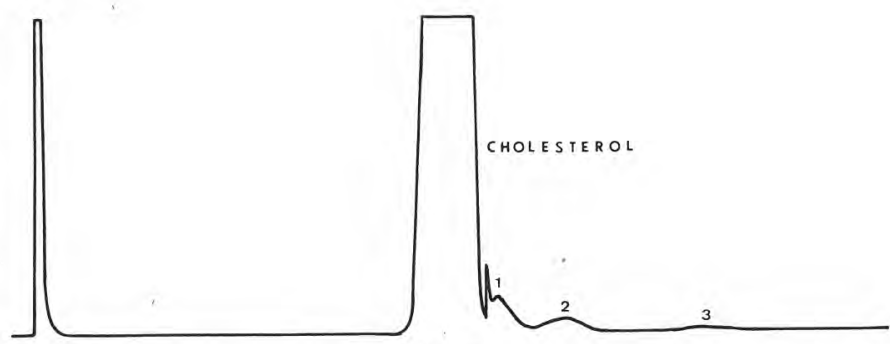

fig. 5

Chromatogramme des stérols d'une matière grasse butyrique

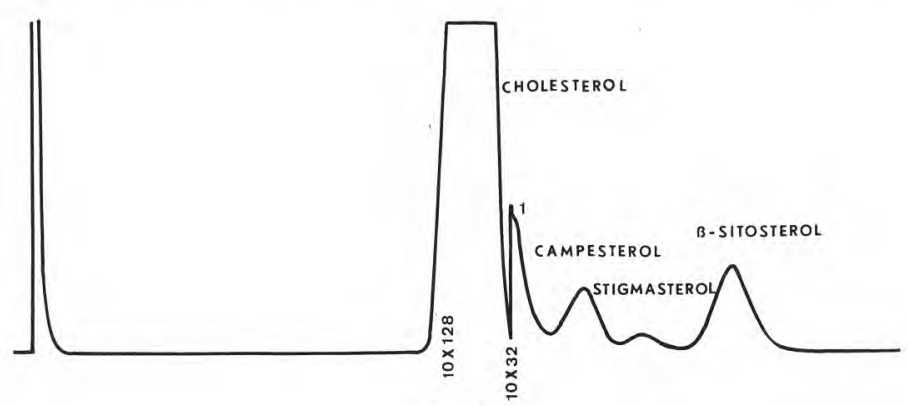

fig. 6

Chromatogramme des stérols d'une graisse butyrique contenant de l'huile de soja 


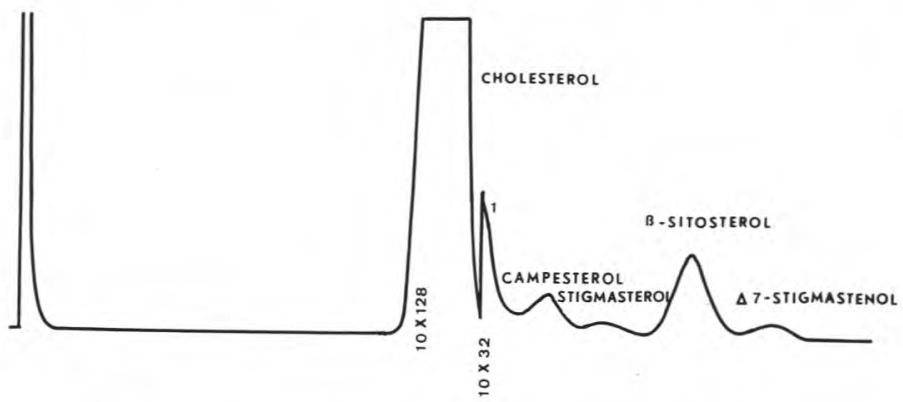

fig. 7

Chromatogramme des stérols d'une graisse butyrique contenant de l'huile de tournesol

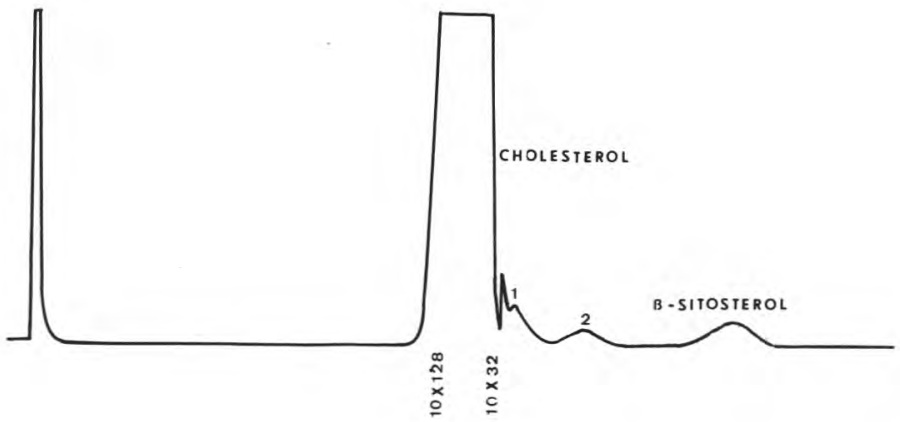

fig. 8

Chromatogramme des stérols d'une graisse butyrique contenant 5 p. 100 d'une graisse butyrique tracée

La proportion relative des stérols peut donner des indications concernant la nature de la graisse végétale. Les figures 6 et 7 démontrent la présence respectivement de l'huile de soja et de l'huile de tournesol. La présence de l'huile de soja est déduite par le rapport $\beta$-sitostérol / stigmastérol; l'huile de tournesol par le $\Delta 7$-stigmastérol.

\section{III. - Les graisses butyriques tracées}

Les méthodes signalées sous 2, permettent aussi de déceler des graisses butyriques tracées à l'aide de $\beta$-sitostérol. Le problème se réduit à une détermination des phytostérols. Comme démontré dans la figure 8 il est possible de déceler des quantités minimes de graisse dénaturée. Il est bien évident que le pourcentage retrouvé est déterminé par la teneur en $\beta$-sitostérol ajouté.

De l'autre côté nous avons également démontré (Hendrickx et Huyghebaert [24]) qu'il est possible par examen des stérols de démontrer des graisses végétales dans la graisse butyrique dénaturée. 
Dans ce cas on ne peut plus tenir compte du $\beta$-sitostérol mais la présence éventuelle de stigmastérol, de brassicastérol ou d'autres stérols végétaux est très importante.

\section{IV. - Les graisses animales}

Les graisses animales, par exemple le saindoux et le suif ne contiennent pas d'acides inférieurs. La présence de ces graisses est démontrée par une diminution de la teneur en acides à chaîne courte et moyenne de $\mathrm{C} 4: 0$ à $\mathrm{C} 12: 0$. Ajoutons ici les possibilités de l'analyse thermique différentielle. Cette technique, basée sur la différence dans les propriétés physiques (courbe de cristallisation), a été proposée par Roos et Tuinstra [25].

La présence éventuelle mais peu probable d'une huile marine est facilement démontrée par les acides à chaîne longue ou dans le cas d'une huile hydrogénée par la présence d'une quantité anormale d'acides trans comme il a été décrit par De Ruig [26].

\section{Conclusion}

Il est évident qu'il existe plusieurs méthodes pour déceler les graisses étrangères dans la matière grasse butyrique.

Pour le problème en général nous retenons trois conclusions :

- Les organisations de contrôle doivent disposer d'une gamme de méthodes d'analyse non seulement pour la détection mais aussi pour la confirmation d'une falsification éventuelle. De préférence ces méthodes doivent être basées sur des propriétés différentes.

- L'exemple des graisses de substitution prouve qu'une vigilance permanente est nécessaire. On peut bien s'imaginer que des types de graisses apparaissent sur le marché qui se rapprochent encore plus de la graisse butyrique.

- Dans l'avenir la détection des fraudes de la graisse butyrique ne sera certainement pas facilitée à la suite des traitements technologiques comme le fractionnement, l'hydrogénation et d'autres qui seront probablement de plus en plus appliqués.

Il n'est donc pas exagéré de conclure que des recherches dans ce domaine doivent se poursuivre.

\section{Résumé}

Les problèmes de détection des différents types de graisses dans la graisse butyrique sont décrites, c'est-à-dire les graisses de substitution ou graisses semblables au beurre, les graisses d'origine végétale et animale et les graisses butyriques tracées. Plusieurs possibilités offertes par des techniques modernes d'analyse comme la chromatographie en phase gazeuse et sur couche mince, l'analyse thermique différentielle et d'autres, particulièrement dans le cas des graisses de substi. 
tution, sont présentées. Il est nécessaire de faire appel à ces techniques modernes parce que les méthodes classiques sont tout à fait insuffisantes pour déceler avec certitude certaines graisses étrangères dans la graisse butyrique.

\section{S u m m a ry}

The detection of foreign fats such as substitute or butterlike fats, vegetable and animal fats and traced butterfats in butterfat is described. The possibilities by modern techniques as gas- and thin layer chromatography, differential thermal analysis and others, especially in the case of substitute fats, are presented. As the classical methods are insufficient, it is necessary to use these methods for the detection of some types of fats in butterfat.

\section{Bibliographie}

[1] Roos (J. B.), FIL. (1963), - Bulletin, n ${ }^{\circ} 4$.

[2] Guillaumin (R.) (1965). - Revue Franç. Corps Gras, 12, 29.

[3] Hopper (J. H.), Gardner (K. F.) and Johnson (B. C.) (1954). - J. Dairy Sci., 37, 431.

[4] Guex (G.). (1957). - Fleischwirtsch, 9, 494.

[5] Bhalerao (V. R.) and Kummerow (F. A.) (1954). - J. Dairy Sci., 37, 156.

[6] Hansen (E.), Sturm (W.) und Von Drachenfels (H.) (1960). - Z. Lebensmitt. Untersuch, 111, 381.

[7] Lücк (H.) und Koнn (R.) (1963). - Fette, Seifen, Anstrichm., 65, 314.

[8] Boniforti (L.) (1962), Ann. Falsif. Expert Chim Paris, 55, 255.

[9] De Francesco (F.) et Avancini (D.) (1961). - Boll. Lab. Chim. prov., 12, 422.

[10] Guyot (A.) et Piraux (E.) (1964). - Bull. Inst. agron. Gembloux, 32, 190.

[11] Roos (J. B.), Versnel (A.) und Werdmuller (G. A.) (1963), - Kieler Milchwirtschaft. Forsch. Berichte, 15, 49.

[12] Parodi (P. W.) (1967). - Aust, J. Dairy Tech., 22, 144.

[13] Parodi (P. W.) (1967). - Aust. J. Dairy Tech., 22, 209.

[14] Roos (J. B.) (1964). - Fette, Seifen, Anstichm., 66, 182.

[15] Hendrickx (H.) en Huyghebaert (A.) (1968). - Meded. Fac. Landb. Gent, $33,331$.

[16] Jensen (R. G.) and Morgan (1959). - J. Dairy Sci., 42, 232.

[17] FIL norme $\mathrm{n}^{\circ}$ 32, 1965.

[18] Guyot (A.) (1968). - Bull. Rech. agro., Gembloux, 3, 128.

[19] Roos (J. B.), Versnel (A.) und VAN DiJK (D. M.) (1969). - Ned. Melk Zuivelt., $23,89$.

[20] Huyghebaert (A.), Hendrickx (H.), Schamp (N.) et De Buyck (L.) (1970). Fette, Seifen, Anstrichm., 72, 289.

[21] LA CRoIX (D.) (1969). - J. Ass. off. Anal. Chem., 52, 600.

[22] Parodi (P. W.) and Dunstan (R. J.) (1968). - Aust. J. Dairy Tech., 23, 20.

[23] Kuzdzal-Savoie (S.) et Kuzdzal (W.) (1969). - Techniq. Lait., n ${ }^{\circ}$ 650, 33.

[24] Hendrickx (H.) en Huyghebaert (A.) (1970), - Meded. Fac. Landb. Gent, 35,65 .

[25] Ross (J. B.) and Tuinstra (L. G. M. I.) (1969). - Ned. Melk Zuivelt, 23, 37.

[26] De Ruig (W. G.) (1968). - Ned. Melk Zuivelt, 22, 104. 